Publ. RIMS, Kyoto Univ.

20 (1984), 867-875

\title{
Real and Complex Monge-Ampère Equations and the Geometry of Strictly Convex Domains
}

By

Giorgio PATRIZIO*

\section{Introduction}

In recent years it has been realized that the Monge-Ampère operator plays an important role in many problems in the theory of several complex variables. In particular, the existence of solutions with certain properties for the homogeneous complex Monge-Ampère equation was used by Stoll [7] to characterize the ball in $\boldsymbol{C}^{m}$. In this note we shall deal with the case of strictly convex domains. For such domains Lempert [5] defined exhaustions which satisfy the complex homogeneous Monge-Ampère equation outside a preassigned point. These exhaustions are closely related to the hyperbolic geometry (in the sense of Kobayashi) of the domain. On the other hand, for strictly convex domains it is also possible to define solutions for the real homogeneous Monge-Ampère equation. The interplay of these solutions gives interesting informations on the classification of the domains. In Sections 1 and 2 we present the results on the real and the complex Monge-Ampère equation which we will use. The main result of Section 1, Theorem 1.2, is due, in an equivalent form, to Foote [2]. Here we give an outline of the proof for the sake of completeness. In Section 3 we give our applications. In Theorem 3.1 we characterize the strictly convex domains which are circular and in Theorem 3.3 we give conditions for a strictly convex domain to be biholomorphic to the ball. Also in Theorem 3.4 we apply our results to the classification theory of tube domains in $C^{m}$ whose base is strictly convex.

We like to thank the referee for his most helpful comments which improved the paper in many points.

Communicated by S. Nakano, August 15, 1983. Revised November 10, 1983.

* The author was supported in part by a fellowship of the Consiglio Nazionale delle Ricerche (Italy).

University of Notre Dame, IN, U.S. A.

SFB 40 Universität Bonn, Fed. Rep. of Germany. 


\section{$\S 0 . \quad$ Notations}

Let $D \subset \boldsymbol{R}^{n}$ be an open, bounded, connected subset. We say that $D$ is a strictly convex domain if there exist a neighborhood $U^{\top}$ of $\partial D$ and a function $r$ : $U \rightarrow \boldsymbol{R}$ of class $C^{\infty}$ such that $U \cap D=\{x \in U \mid r(x)<0\}$ and $\partial D=\{x \in U \mid r(x)=0\}$ with $d r(x) \neq 0$ if $x \in \partial D$ and the property that the real Hessian $\mathrm{H}_{r}$ of $r$ is positive definite.

We shall work with domains in $\mathbb{C}^{m}$ and real and complex derivatives will appear. Whenever possible we shall simplify the notations as follows. If $f$ is a function of class $\mathrm{C}^{k}$ on an open subset of $\mathbf{C}^{m},\left(x^{1}, \cdots, x^{2 m}\right)$ are real coordinates and $\left(z^{1}, \cdots, z^{m}\right)$ are complex coordinates, then latin subscripts will denote real derivatives and greek subscripts will denote complex derivatives:

$$
\begin{aligned}
& f_{J_{1} \cdots j_{s}}=\frac{\partial}{\partial x^{\jmath_{1}}} \cdots \frac{\partial}{\partial x^{\jmath_{s}}} f \quad \text { for } s \leqq k, \\
& f_{\mu_{1} \cdots / p^{\prime} \bar{\nu}_{1} \cdot \bar{\nu}_{q}}=\frac{\partial}{\partial z^{\mu_{1}}} \cdots \frac{\partial}{\partial z^{\mu} p} \frac{\partial}{\partial \bar{z}^{\nu_{1}}} \cdots \frac{\partial}{\partial \bar{z}^{\nu} q} f \text { for } \quad p+q \leqq k .
\end{aligned}
$$

Also Einstein's summation convention will be used if no confusion arises. In this notation the real Hessian form for a function $r$ of class $\mathrm{C}^{2}$ on an open set $U \subset \mathbb{R}^{n}$ at $p \in U$ for $X, Y \in \boldsymbol{R}^{n}$ is given by :

$$
\mathrm{H}_{r}(p, X, Y)=r_{j k}(p) X^{\jmath} Y^{k},
$$

and the Levi form for a function $u$ of class $C^{2}$ on an open set $V \subset C^{m}$ at $q \in V$ for $Z, W \in C^{m}$ is given by :

$$
\mathrm{L}_{u}(q, Z, W)=u_{\mu \bar{\nu}}(q) Z^{\mu} \overline{W^{\nu}} .
$$

We also define the real Monge-Ampère operator for $r$ by

$$
\mathrm{N}_{R}(r)=\operatorname{det}\left(r_{j k}\right),
$$

and the complex Monge-Ampère operator for $u$ by

$$
\mathrm{N}_{C}(u)=\operatorname{det}\left(u_{\mu \bar{\nu}}\right) \text {. }
$$

\section{$\S 1$. The Real Case}

Let $D \subset \boldsymbol{R}^{n}$ be a strictly convex domain and $p \in D$. Denote $D_{*}=D-\{p\}$ and $\bar{D}_{*}=\bar{D}-\{p\}$. A proper continuous function $r: \bar{D} \rightarrow[0,1]$ is called a radial exhaustion of $D$ with center $p$ if it satisfies the following assumptions:

(1.1) $r(p)=0, r \equiv 1$ on $\partial D$ and $0<r<1$ on $D_{*}$,

(1.2) $r$ is of class $\mathrm{C}^{3}$ on $\bar{D}_{*}$,

(1.3) if $R=r^{2}$ then $\mathrm{H}_{R}>0$ on $D_{*}$. 
(1.4) $\mathrm{N}_{R}(r) \equiv 0$ on $D_{*}$.

Lemma 1.1. Let $G \subset \boldsymbol{R}^{n}$ be an open set and $r: G \rightarrow \boldsymbol{R}$ be a function of class $\mathrm{C}^{2}$. If $R=r^{2}$ is such that $\mathrm{H}_{R}>0$ then the following statements are equivalent

(1.5) $\mathrm{N}_{R}(r) \equiv 0 \quad$ on $\quad G$,

(1.6) $\operatorname{rank}_{R}\left(r_{j k}\right)=n-1$ on $G$,

(1.7) $\quad R_{\jmath} R^{j k} R_{k}=2 R \quad$ on $G$ where we set $\left(R^{\jmath k}\right)=\left(R_{\jmath k}\right)^{-1}$.

Proof. The proof is an easy calculation and we give here just an outline. Clearly $(1.6) \Rightarrow(1.5)$. Let $T=\mathrm{N}_{R}(R)$ and let $T^{j k}$ be the minor determinant associated to $R_{j k}$. Then since $4 R^{3 / 2} r_{j k}=2 R R_{j k}-R_{j} R_{k}$, using some matrix manipulations one gets

$$
\left(4 R^{3 / 2}\right)^{n} \mathrm{~N}_{R}(r)=(2 R)^{n-1}\left(2 R T-R_{\jmath} T^{\jmath k} R_{k}\right)
$$

which proves $(1.5) \Leftrightarrow(1.7)$. On the other hand, since $R_{\jmath k}=2\left(r r_{j k}+r_{j} r_{k}\right)$, if we denote $t^{j k}$ the minor determinant associated to $r_{\jmath k}$, we obtain $0 \neq T=2^{n} r^{n-1} r_{j} t^{i k} r_{k}$ which shows $(1.5) \Rightarrow(1.6)$. q.e.d.

If as above $D \subset \mathbb{R}^{n}$ is a strictly convex domain and $p \in D$, we define the Minkowski functional $m_{p}$ of $D$ at $p$ in the following way:

$$
\left\{\begin{array}{l}
m_{p}(p)=0 \\
m_{p}(q)=\inf \{1 / t \mid t>0 \text { and } p+t(q-p) \notin D\} \text { if } q \neq p .
\end{array}\right.
$$

Since we assume $\partial D$ to be smooth of class $C^{\infty}, m_{p}$ is proper and continuous on $\boldsymbol{R}^{n}$ and of class $\mathrm{C}^{\infty}$ on $\boldsymbol{R}^{n}-\{p\}$ (cf. [8]). We also set

$$
M_{p}=m_{p}^{2} .
$$

Theorem 1.2. Let $D \subset \boldsymbol{R}^{n}$ be a strictly convex domain and $p \equiv D$ be any point. Then $\left.m_{p}\right|_{\bar{D}}$ is a radial exhaustion of $D$ with center at $p$. Conversely if $r: \bar{D} \rightarrow$ $[0,1]$ is a radial exhaustion of $D$ with center at $p$, then $r=\left.m_{p}\right|_{\bar{D}}$.

Proof. Put $m=m_{p}$ and $M=M_{p}$ and assume, without loss of generality, that $p=0$. Trivially (1.1) and (1.2) hold for $m$. Let $q \in D-\{0\}$. Then, since $m(t q)$ $=\operatorname{tm}(q)$ for all $t \geqq 0$ we have by differentiation

$$
m_{j}(q) q^{j}=m(q)>0,
$$

so that $m_{j k}(q) q^{j}+m_{k}(q)=m_{k}(q)$ and hence

$$
m_{j k}(q) q^{j}=0,
$$

from which it follows that $m$ satisfies (1.4). Let $s=m(q)$ and define $D_{s}$ $=\{x \in D \mid m(x)<s\}$. Then $D_{s}$ is strictly convex. Since the tangent space of $\partial D_{s}$ at $q$ is given by 


$$
\mathrm{T}_{q}\left(\partial D_{s}\right)=\left\{Y \in \boldsymbol{R} \mid m_{\jmath}(q) Y^{j}=0\right\},
$$

(1.10) implies $q \oplus \mathrm{T}_{q}\left(\partial D_{s}\right)$ and hence $\boldsymbol{R}^{n}=\boldsymbol{R} q+\mathrm{T}_{q}\left(\partial D_{s}\right)$. Let $X \in \boldsymbol{R}^{n}-\{0\}$. Then $t \in \boldsymbol{R}$ and $Y \in \mathrm{T}_{q}\left(\partial D_{s}\right)$ exist uniquely so that $X=t q+Y$. As $M_{j k}=2\left(m m_{j k}+m_{j} m_{k}\right)$, we have using (1.10) and (1.11)

$$
\begin{aligned}
\mathrm{H}_{M}(q, X, X) & =M_{\jmath k}(q) X^{\jmath} X^{k} \\
& =2\left(s m_{\jmath k}(q) Y^{\jmath} Y^{k}+\left(m_{\jmath}(q) t q^{\jmath}\right)\left(m_{k}(q) t q^{k}\right)\right) \\
& =2\left(s \mathrm{H}_{m}(q, Y, Y)+s^{2} t^{2}\right),
\end{aligned}
$$

so that if $Y \neq 0$ then $\mathrm{H}_{m}(q, Y, Y)>0$ since $D_{s}$ is strictly convex, if $Y=0$ then $t>0$ and hence $\mathrm{H}_{M}(q, X, X)>0$ in either case. This shows that $m$ satisfies (1.3) and hence $m$ is a radial exhaustion of $D$ with center $p$.

Conversely assume that $r$ is a radial exhaustion of $D$ with center $p$. We can assume $p=0$. Define $R=r^{2}$ and let $\left(R^{j k}\right)=\left(R_{\jmath_{k}}\right)^{-1}$. A vector field $\xi: \bar{D}_{*} \rightarrow \boldsymbol{R}^{n}$ of class $\mathrm{C}^{1}$ is defined by

$$
\xi^{\jmath}=\frac{R^{\jmath k} R_{k}}{r}=2 R^{\jmath k} r_{k}
$$

so that

$$
\begin{aligned}
& R_{j k} \xi^{j}=2 r_{k}, \\
& R_{j k a} \xi^{j}+R_{j k} \xi_{a}^{j}=2 r_{k a} .
\end{aligned}
$$

Using (1.7) we have

$$
r_{\jmath} \xi^{j}=\frac{R_{j} R^{j k} R_{k}}{2 R}=1 \text { and } R_{\jmath} \xi^{j}=2 r .
$$

Then, since $2 r r_{j} \xi^{\jmath}=R_{j k} \xi^{\jmath}-2 r_{j} \xi^{\jmath} r_{k}=2 r_{k}-2 r_{k}=0$, we have

$$
r_{j k} \xi^{j}=0 \text {. }
$$

From (1.13) and (1.14) it follows that $R_{\jmath_{k}} \xi^{j} \xi^{k}=2$ and hence differentiating

$$
R_{j k a} \xi^{\jmath} \xi^{k}+R_{\jmath k} \xi_{a}^{j} \xi^{k}+R_{\jmath k} \xi^{\jmath} \xi_{a}^{k}=0 .
$$

Therefore using (1.13) and (1.15)

$$
R_{\jmath k} \xi^{j} \xi_{a}^{k}=0 \text { and } \quad R_{\jmath k a} \xi^{\jmath} \xi^{k}=0 .
$$

Also from (1.13) we have

$$
R_{j k} \xi_{a}^{j} \xi^{a}+R_{\jmath k a} \xi^{j} \xi^{a}=2 r_{k a} \xi^{a}=0,
$$

so that using (1.17)

$$
R_{\jmath k} \xi_{a}^{j} \xi^{a}=0 .
$$

Since $\left(R,_{k}\right)$ is invertible, we conclude from (1.19) 


$$
\xi_{a}^{j} \xi^{a}=0 \quad \text { for all } j=1, \cdots, n .
$$

Because of (1.14) there exists a flow $\dot{\phi}:(0,1] \times \bar{D}_{*} \rightarrow \bar{D}_{*}$ such that for all $x \in \bar{D}_{*}$ and $t \in(0,1]$

$$
\begin{aligned}
& \dot{\phi}(t, x)=\xi(\phi(t, x)) . \\
& \dot{\phi}(1, x)=\frac{x}{m(x)} .
\end{aligned}
$$

But then

$$
-\frac{d r}{d t}(\dot{\phi}(t, x))=r_{\jmath}(\dot{\phi}(t, x)) \hat{\xi}^{\jmath}(\phi(t, x))=1
$$

and hence for all $x$ there exists a constant $c(x)$ such that $r \dot{\varphi} t . x))=t+c(x)$. Since $1=r\left(-\frac{x}{m(x)}\right)=r(\phi(1, x))=1+c(x)$, we have $c(x)=0$ for all $x$ and

$$
r(\dot{\phi}(t, x))=t .
$$

Also by (1.20) and (1.21) we have

$$
\ddot{\phi}(t, x)=\xi_{k}(\phi(t, x)) \xi^{k}(\dot{\phi}(t, x))=0 .
$$

Then there are vectors $\phi(x)$ and $\chi(x)$ so that

$$
\phi(t, x)=\phi(x)+t \chi(x) .
$$

But, since $r(\dot{\phi}(x)+t \chi(x))=r(\dot{\phi}(t, x))=t$, from (1.22) taking limit as $\rightarrow 0$ we get $r(\phi(x))=0$ i. e. $\phi(x)=0$. Then (1.23), for $t=1$, gives $\chi(x)=\begin{gathered}r \\ n l\end{gathered}$, Hence $\dot{\phi}(m(x), x)=m(x) \chi(x)=x$ and

$$
m(x)=r(\phi(m(x), x))=r(x) . \quad \text { q. e. d. }
$$

Corollary 1.3. Let $D \subset \boldsymbol{R}^{n}$ be a strictly convex domain. If here exists a radial exhaustion $r: \bar{D} \rightarrow[0,1]$ of $D$ such that $R=r^{2}$ is of class $C^{-}$at the center $p$ of $r$, then $R$ is a positive definite quadratic form and there exists $A \equiv \mathrm{GL}(n, \boldsymbol{R})$ such that $D=\mathcal{A}(\boldsymbol{B})+p$ where $\boldsymbol{B}$ is the unit ball in $\boldsymbol{R}^{n}$.

Proof. Without loss of generality we can assume that $p=0$. Let $m$ be the Minkowski functional of $D$ at the center $p=0$. If $M=m^{2}$ then $R=\left.M\right|_{\bar{D}}$ and hence for all $x \in D$ and $t \in \boldsymbol{R}$ small enough we have $\left.R(t, x)=\mid I^{\prime} t x\right)=t^{2} M(x)$ $=t^{2} R(x)$. Thus

$$
2 R(x)=\frac{\partial^{2}}{\partial t^{2}} R(t x)=\mathrm{H}_{R}(t x, x, x),
$$

and hence $R(x)=\frac{1}{2} \mathrm{H}_{R}(0, x, x)$ and the result follows.

q.e.d.

Remark. Corollary 1.3, which is a real analogue of Stoll's characterization 
of the ball in $C^{n}([7])$, has been proved in the more general setting of affine manifolds by Burns [1] and Foote [2] under a little stronger differentiability assumption on $r$.

\section{$\S 2$. The Complex Case}

An explicit solution for the complex Monge-Ampère equation $\mathrm{N}_{C}(u)=0$ on a strictly convex domain outside a preassigned point was given by Lempert [5]. Applications to the theory of parabolic manifolds are given in [6]. Here we limit ourselves to definitions and statements of the basic properties.

Let $D \subset C^{n}$ be a strictly convex domain and $p \in D$ be any point. If $J=$ $\{z \in C|| z \mid<1\}$ is the unit disk in $\boldsymbol{C}$ and $\boldsymbol{S}$ is the unit sphere in $\mathbf{C}^{m}$, then there exists uniquely a surjective map $F: \bar{J} \times \boldsymbol{S} \rightarrow \bar{D}$ of class $\mathrm{C}^{\infty}$ with the following properties :

(2.1) $\quad F(\square, b): \bar{J} \rightarrow \bar{D}$ is proper of class $C^{\infty}$ and $F(\square, b): \Delta \rightarrow D$ is holomorphic with $F\left(0, b^{\prime}=p\right.$ and $F^{\prime}(0, b)=\left\|F^{\prime}(0, b)\right\| b$ for all $b \in S$.

(2.2) A function $P: \partial \Delta \times S \rightarrow(0, \infty)$ of class $C^{1 / 2}$ exists so that the map $\tilde{F}: \partial \Delta$ $\times S \rightarrow C^{m}$ defined by $\tilde{F}(z, b)=z P(z, b) \overline{n(F(z, b))}$, where $n$ denotes the euclidean normal to $\partial D$, extends continuously to a map $\tilde{F}: \Delta \times S \rightarrow C^{m}$ with $\tilde{F}(\sqsubset, b): \Delta \rightarrow C^{m}$ holomorphic for all $b \in \boldsymbol{S}$.

The map $F$ is related to the hyperbolic geometry of $D$ in the following way. Let $K$ be the Kobayashi metric on $D$ and for $q \in D$ denote by $d(q)$ the Kobayashi distance from $p$ to $q$. Then identifying $S$ with the unit sphere in the tangent space we have:

$$
K(p, b)=-\frac{1}{F^{\prime}(0, b) \|}>0 \quad \text { for all } b \in S
$$

(2.4) $d(q)=\frac{1}{2} \log \frac{1+|z|}{1-|z|}$ for all $q \in D$ where $q=F(z, b)$ for some $(z, b) \in \Delta \times S$.

Using the fact that $F(\square, b)$ is the unique holomorphic map which satisfies (2.3), it is easy to show that if $\lambda \in \partial \mathcal{A}$ then $F(z, \lambda b)=F(\lambda z, b)$ for all $(z, b) \in \bar{\Delta} \times \mathbb{S}$. Moreover if $b_{1}, b_{2} \in S$ and $L_{j}=F\left(\square, b_{j}\right)(\bar{d})$ for $j=1,2$, then either $L_{1} \cap L_{2}=\{\not\}$ or $L_{1}=L_{2}$ and there exists $\mu \in \partial \Delta$ with $b_{1}=\mu b_{2}$. A function $\tau=\tau_{p}: \bar{D} \rightarrow[0,1]$. called the Lemport exhaustion of $D$ at $p$, is well defined by

$$
\tau_{p}(q)=|z|^{2} \text { if } q=F(z, b) \text { for some }(z, b) \in \bar{J} \times \mathcal{S} \text {. }
$$

Theorem 2.1. Let $D \subset C^{m}$ be a strictly convex domain and $p \in D$ be any point. Then the Lempert exhaustion $\tau$ at $p$ has the following properties:

(i) $\tau$ is continuous and proper, of class $\mathrm{C}^{\infty}$ on $\bar{D}-\{p\}$ with $\tau(p)=0,0<\tau(q)<1$ if $q \in D-\{p\}$ and $\tau \equiv 1$ on $\partial D$.

(ii) $\mathrm{L}_{\tau}>0$ on $D-\{p\}$. 


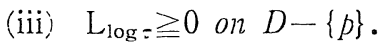

(iv) $\mathrm{N}_{C}(\log \tau)=0$ on $D-\{p\}$.

Proof of (i), (iii) and (iv) is in [5] and (ii) is explicitly prored in [6].

\section{§3. Applications to the Classification of Domains}

In this section we will assume the notations developed in the previous two sections. A domain $G \subset \mathbf{C}^{m}$ is said to be circular if there exists $z_{0} \subseteq G$, called the center of $G$, such that if $\lambda \in C$ with $|\lambda|=1$ and $z \in G$ then $\lambda\left(z-z_{0}\right)+z_{0} \in G$. We have the following characterization of the strictly convex domains which are circular.

Theorem 3.1. Let $D \subset C^{m}$ be a strictly' convex domain. Then $D$ is circular if and only if there exists a point $p \in D$ with $M_{p}=\tau_{p}$.

Proof. Assume $D$ is circular and let $p$ be its center. Without loss of generality we can assume $p=0$. Set $M=M_{p}$ and $\tau=\tau_{p}$. To show $M=\tau$ it will be enough to prove that the map $F: \bar{J} \times S \rightarrow \bar{D}$ defined by $F(z, b)=z m(b)^{-1} b$ satisfies (2.1) and (2.2). Clearly (2.1) holds for $F$. Define $P: \partial \downarrow \times S \rightarrow 0,>)$ by $2 P(z, b)$ $=\|\nabla M(F(z, b))\|$ where $\nabla M$ denotes the euclidean gradient of $M$. Then, if $\tilde{F}: \partial \Delta \times \boldsymbol{S} \rightarrow \boldsymbol{C}^{m}$ is defined by $\left.\tilde{F}(z, b)=z P(z, b) \overline{n(F(z, b)}\right)$, we have $\tilde{F}^{\mu}(z, b)$ $=\frac{M_{1 \prime}\left(z m(b)^{-1} b\right)}{\bar{z}}$. Since $D$ is circular, for all $z \in C^{m}$ and $z \equiv C$ we have $M(z Z)$ $=\left.{ }_{1} z\right|^{2} M(Z)$ and hence $M_{\mu l}(z Z)=\bar{z} M_{\mu}(Z)$. But then $\left.\left.\tilde{F}^{\mu}(z, b)=\mid Y_{n}: m l^{\prime} b\right)^{-1} b\right)$ is constant for all $\mu$ and thus $F$ satisfies (2.2) too.

Conversely, let $M_{p}=\tau_{p}$ for some $p \in D$. Without loss of generality we can assume $p=0$ and set $M=M_{0}$ and $\tau=\tau_{0}$. If $F: \bar{J} \times \boldsymbol{S} \rightarrow \bar{D}$ is the map defined by (2.1), (2.2) for $D$ at 0 , then given any $t \in(0,1]$ and $b \in S$ we have $M(F(t, b))=t^{2}$ and thus $\frac{F(t, b)}{t} \in \partial D$. Then also $F^{\prime}(0, b) \in \partial D$ for all $b \in S$. Let $w \in \partial D$ and $c=w /\left\|u u^{\prime}\right\|$. Then $F^{\prime}(0, c) \in \partial D$ and

$$
F^{\prime}(0, c)=\left\|F^{\prime}(0, c)\right\| c=\frac{\left\|F^{\prime}(0, c)\right\|}{\|w\|} w .
$$

Since $M\left(F^{\prime}(0, c)\right)=M(w)=1$, we have $\left\|F^{\prime}(0, c)\right\|=\|u\|$ and $u=F \quad$ U. $\iota$. . We conclude $\partial D=\left\{F^{\prime}(0, b) \mid b \in \boldsymbol{S}\right\}$. But then

$$
\begin{aligned}
D & =\left\{z \in \boldsymbol{C}^{m} \mid z=t w \text { with } t \in[0,1) \text { and } w \in \partial D\right\} \\
& =\left\{z \in \boldsymbol{C}^{m} \mid z=t F^{\prime}(0, b) \text { with } t \in[0,1) \text { and } b \subseteq S\right\} .
\end{aligned}
$$

Therefore if $Z \in D$ with $Z=t F^{\prime}(0, b)$ and $\lambda \in C$ with $\mid \lambda i=1$, then, since $F(\lambda z, b)$ $=F(z, \lambda b)$ for all $(z, b) \in \Delta \times S, \lambda Z=\lambda t F^{\prime}(0, b)=t F^{\prime}(0, \lambda b) \in D$ and $D$ is circular.

q.e.d.

Because of (2.4), Theorem 3.1 has the following interesting reformulation. 
Theorem 3.1'. A strictly convex domain $D \subset C^{m}$ is circular if and only if there exists $p \equiv D$ such that

$$
d(q)=\frac{1}{2} \log \frac{1+m_{p}(q)}{1-m_{p}(q)}
$$

is the Kobayashi distance from $p$ to $q$ for all $q \in D$.

Using Theorem 1.2 it is also possible to characterize in terms of the real Monge-Ampère equation when a strictly convex domain is circular.

Theorem 3.2. Let $D \subset C^{m}$ be a strictly convex domain. Then $D$ is circular if and only if there exists a point $p \in D$ such that the Lempert exhaustion $\tau=\tau_{p}$ of $D$ at $p$ satisfies $\mathrm{N}_{R}(\sqrt{\tau})=0$ on $D_{*}=D-\{p\}$.

Proof. In light of Theorem 2.1 and Theorem 3.1, we have only to show that if there exists $p \in D$ so that the associated Lempert exhaustion $\tau$ satisfies $N_{R}(\sqrt{\tau})=0$, then $r=\sqrt{\tau}$ is a radial exhaustion for $D$. Since $\tau$ satisfies (1.1), (1.2), (1.4), only $H_{s}>0$ has to be proved. Let $q \in D$ and define $s=r(q)$. In [6] it is shown that $D_{s}=\left\{x \in D \mid \tau(x)<s^{2}\right\}=\{x \in D \mid r(x)<s\}$ is strictly convex. Then it follows that $\mathrm{H}_{r}$ is strictly positive on $\mathrm{T}_{q}(\partial D)$ and $\operatorname{rank}_{R}\left(r_{j_{k}}\right)=2 m-1$. If $0 \neq X$ $\in \boldsymbol{R}^{2 m}=\boldsymbol{C}^{m}$, we can choose $Y \in \mathrm{T}_{q}(\partial D)$ and $V$ tangent at $q$ to the foliation generated by $r$ so that $X=V+Y$. Then at $q$

$$
\mathrm{H}_{\tau}(X, X)=2\left(r_{\jmath} r_{k} V^{j} V^{k}+s \mathrm{H}_{r}(Y, Y)\right)>0 . \quad \text { q. e. d. }
$$

Using the above results we can give the following characterization of the unit ball $B \subset C^{m}$.

Theorem 3.3. Let $D \subset C^{m}$ be on strictly convex domain. Then we have the following facts.

(i) Assume there exists $p \in D$ such that $m_{p}$ is of class $C^{2}$ at $p$. Then $D$ is biholomorphic to $\boldsymbol{B}$ if and only if $M_{p}=\tau_{p}$.

(ii) Assume there exists $p \in D$ such that $m_{p}$ is of class $C^{2}$ at $p$. Then $D$ is biholomorphic to $B$ if and only if $\mathrm{N}_{R}\left(\sqrt{\tau_{p}}\right)=0$ on $D_{*}=D-\{p\}$.

(iii) Assume there exists $p \in D$ such that $\tau_{p}$ is of class $C^{2}$ at $p$. If $\mathrm{N}_{\boldsymbol{R}}\left(\sqrt{\tau_{p}}\right)$ $=0$ on $D_{*}$, then $D$ is biholomorphic to $\boldsymbol{B}$.

Proof. (i Because of Corollary 1.3, if $m_{p}$ is of class $C^{2}$ then $D$ is an ellipsoid. By a theorem of Webster [9], then $D$ is biholomorphic to $B$ if and only if it is circular. Theorem 3.1 gives then the conclusion.

(ii) The same proof of (i) together with Theorem 3.2 yields (ii).

(iii) If $\tau_{2}$, is of class $\mathrm{C}^{2}$ and $\mathrm{N}_{R}\left(\sqrt{\tau_{p}}\right)=0$ then Theorem 3.2 and Corollary 1.3 imply that $D$ is a circular ellipsoid and hence biholomorphic to $\boldsymbol{B}$. q.e.d.

Another application of Theorem 1.2 is in the classification theory of tube domains in $C^{m}$. Let $D$ be a strictly convex domain in $\boldsymbol{R}^{m}$. The tube domain $\Phi(D) \subset C^{m}$ uith base $D$ is defined by $q(D)=D+i \boldsymbol{R}^{m}$. Denote the Minkowski 
functional of $D$ at $p \in D$ by $m_{D, p}$. We have the following.

Theorem 3.4. Let $D, D^{\prime} \subset \boldsymbol{R}^{m}$ be two strictly convex donains. These two facts are equivalent:

(i) $I(D)$ is biholomorphic to $I\left(D^{\prime}\right)$.

(ii) There exists $p \in D, q \in D^{\prime}$ and an affine isomorphism $A$ of $R^{m}$ such that $m_{D, p}=m_{D^{\prime}, q^{\circ}} A$.

Proof. Assume that (i) is true. We can assume that $0 \in D$. By [4] and [10] it follows that $I(D)$ and $I\left(D^{\prime}\right)$ are affinely equivalent and therefore there exists an affine isomorphism $A$ of $\boldsymbol{R}^{m}$ such that $A(D)=D^{\prime}$. If $A(0)=q$ then $A$ $=q+B$ where $B \in \mathrm{GL}(\boldsymbol{R}, m)$. Define $r=m_{D^{\prime}, q^{\circ}} A$. Then $r$ is a radial exhaustion of $D$ with center 0 and hence, by Theorem 1.2, $r=m_{D, 0}$ i.e. (ii) holds.

Conversely assume that (ii) is true. Then, since $m_{D, p}(x)=0$ if and only if $x=p$ and $m_{D^{\prime}, q}(x)=0$ if and only if $x=q$, we have $A(x)=q+B(x-p)$ where $B$ $\in \mathrm{GL}(\boldsymbol{R}, m)$. Then the map $L: \boldsymbol{C}^{m} \rightarrow \boldsymbol{C}^{m}$ defined by $L(z)=L(x+i y)=q+B(x-p)$ $+i B(y)$ gives a biholomorphic map between $\mathscr{I}(D)$ and $\mathscr{I}\left(D^{\prime}\right)$. q.e.d.

\section{References}

[1] Burns, D., Curvature of Monge-Ampère foliations and parabolic manifolds, Ann. of Math., 115 (1982), 349-373.

[2] Foote, R.L., Curvature estimates for Monge-Ampère foliations, University of Mi. chigan Thesis, 1983.

[3] Hartman, P. and Nirenberg, L., On spherical image maps whose Jacobians do not change sign, Amer. J. of Math., 81 (1959), 901-920.

[4] Kaup, W., Matsushima, Y. and Ochiai, T., On the automorphisms and equivalences of generalized Siegel domain, Amer. J. of Math., 92 (1970), 475-498.

[5] Lempert, L., Le métrique de Kobayashi et la représentation des domains sur la boule, Bull. Soc. Math. de France, 109 (1981), 427-474.

[6] Patrizio, G., Parabolic exhaustions for strictly convex domains, manuscripta math., 47 (1984), 271-309.

[7] Stoll, W., The characterization of strictly parabolic manifolds, Ann. Scuola Norm. Sup. Pisa, 7 (1980), 87-154.

[8] Stout, E. L., On the multiplicative Cousin problem with bounded data, Ann. Scuola Norm. Sup. Pisa, XXVII (1973), 1-17.

[9] Webster, S. M., On the mapping problem for algebraic real hypersurfaces, Inventiones Math., 43 (1977), 53-68.

[10] Yang, P.C., Automorphisms of tube domains, Amer. J. of Math., 104 (1982), 10051024. 
